\title{
Methyl Palmitate: the Naturally Occurring Cardioprotective Agent
}

\author{
Ahmed B. Hamed ${ }^{* a}$, Eman M. Mantawy ${ }^{b}$, Wesam M. El-Bakly ${ }^{c}$, Yousra Abdel-Mottaleb ${ }^{\text {a }}$, Samar S. Azab \\ ${ }^{a}$ Pharmacology, Toxicology and Biochemistry Department, Faculty of Pharmaceutical Sciences and Pharmaceutical \\ Industries, Future University in Egypt, Cairo 11835, Egypt \\ ${ }^{\mathrm{b}}$ Pharmacology and Toxicology Department, Faculty of Pharmacy, Ain shams University, Cairo 11566, Egypt \\ ${ }^{\mathrm{c}}$ Pharmacology and Therapeutics Department, Faculty of Medicine, Ain Shams University, Cairo, Egypt
}

\begin{abstract}
Cardioprotective agents are compounds that provide heart protection and decrease cardiotoxicity incidence. Cardiotoxicity is a serious condition that results in diminishing the heart's ability to pump blood throughout the body and can be developed into heart failure. Oxidative stress is an important pathogenic event in cardiotoxicity where the generated reactive oxygen species (ROS) cause myocardial cellular destruction. Moreover, apoptosis, fibrosis, and inflammatory cascades play major roles in cardiotoxicity pathogenesis. Recently, much attention has been paid to the cardioprotective effects of natural products. Methyl palmitate (MP) is a naturally occurring methyl ester that can be also synthesized. This review article aimed to elucidate the potential cardioprotective effects of MP as well as the underlying possible mechanisms. Indeed, MP showed effective cytoprotective roles in various experimental models. In this regard, MP showed potent antioxidant activity which was proven by the decreased production of oxidative stress markers and the increased activity of the endogenous antioxidant enzymes. It also exhibited anti-inflammatory activity which was evidenced by the reduced expression of the pro-inflammatory cytokines and the elevated expression of the anti-inflammatory cytokines. Moreover, MP showed anti-apoptotic activity evidenced by the elevated anti-apoptotic protein expression and the mitigated pro-apoptotic protein expression. Besides, further studies proved the anti-fibrotic and vasodilatation activities of MP. Thus, MP could provide major cardioprotective activities through its antioxidant, anti-inflammatory, anti-apoptotic, anti-fibrotic, and vasodilatation properties.
\end{abstract}

Keywords: Cardioprotection; Methyl palmitate; Antioxidant; Anti-inflammatory; Anti-apoptotic; Anti-fibrotic; Vasodilatation.

*Correspondence | Ahmed Hamed; Pharmacology, Toxicology and Biochemistry Department, Faculty of Pharmaceutical Sciences and Pharmaceutical Industries, Future University in Egypt, Cairo 11835, Egypt. Email: ahmed.badreldin@fue.edu.eg

Citation | Hamed AB, Mantawy EM, El-Bakly WM, Abdel-Mottaleb Y, Azab SS, 2020. Methyl palmitate: the naturally occurring

cardioprotective agent. Arch Pharm Sci ASU 4(1): 47-62

DOI: $10.21608 /$ APS.2020.2003.1026

Print ISSN: 2356-8380. Online ISSN: 2356-8399.

Received 17 April 2020. Accepted 16 May 2020.

Copyright: ${ }^{\circ} 2020$ Hamed et al. This is an open-access article licensed under a Creative Commons Attribution 4.0 International License (CC BY 4.0), which permits unrestricted use, distribution, and reproduction in any medium, provided the original author(s) and source are credited.

Published by: Ain Shams University, Faculty of Pharmacy

\section{INTRODUCTION}

Cardioprotective agents are compounds that protect cardiac muscle by reducing or even preventing cardiotoxicity occurrence [1].
Cardiotoxicity is a critical condition that is characterized by cardiac electrophysiological dysfunction or cardiac muscle damage. It may result in the weakening of the cardiac muscle and diminishing of blood pumping efficacy which 
may progress into heart failure [2]. Cardiotoxicity may result from different factors such as chemotherapy treatment, chronic alcohol consumption, smoking, or as an adverse effect of heavy metals intake [3]. Accordingly, there is a massive need for exploring natural products with promising cardioprotective effects [4].

Methyl palmitate (MP; Hexadecanoate methyl ester; Methyl ester of palmitic acid) is a naturally occurring fatty acid ester [5]. It has a polar carboxylate group and nonpolar aliphatic radical with the empirical formula $\mathrm{C}_{17} \mathrm{H}_{34} \mathrm{O}_{2}$ and a molecular weight of 270.45. Besides, MP is a white crystalline water-insoluble powder that can be readily soluble in oil [6]. It was proven that MP is released in the superior cervical ganglion [7] and the retina [8] of rats. Moreover, MP is a naturally occurring botanical compound that can be found in many plants, such as the bark of Tonka bean and Dipteryx odorata aubl and fruits of Ficus carica L. [9]. Additionally, it can be also found in the leaves of Daphne genkwa sieb. et zucc, mangrove and Salvadora persica L., flowers of mangrove and Salvadora persica $L$. and stems of the Chateau and Delavaya yunnanensis Franch [10]. Furthermore, MP can be easily synthesized via a condensation reaction between methyl alcohol and palmitic acid [11]. It is known that MP is safe for vertebrates, as reflected by being widely used in food, pharmaceuticals, industrial applications, and cosmetics [10].

Most importantly, MP has been recently reported to have cytoprotective roles in various experimental models [12, 13]. These cytoprotective effects mainly attributed to its potent antioxidant, anti-inflammatory, antiapoptotic, anti-fibrotic, and vasodilatation activities [14, 15]. Thus, the present article aims to elucidate the possible cardioprotective activities of MP as well as the underlying molecular mechanisms.

\section{CARDIOTOXICITY RISK FACTORS}

\subsection{Chemotherapy treatment}

Chemotherapy treatment is considered a major causative factor of cardiotoxicity which can be early or lately appeared during the treatment. Subclinical myocardial dysfunction, irreversible heart failure, or even death are the major outcomes of chemotherapy treatment [16].

Doxorubicin (DOX) is one of the most effective chemotherapeutic agents that have dosedependent cardiotoxic effects. Indeed, DOX can induce either acute cardiotoxicity, shortly after treatment initiation, or late-onset chronic cardiotoxicity [17]. Myocardial oxidative stress plays a major role in DOX-induced cardiotoxicity. In this context, DOX is reported to produce massive amounts of reactive oxygen species (ROS) within the myocardium, resulting in increased lipid peroxidation and cardiomyocytes damage. Besides, it also induces both the intrinsic and the extrinsic apoptotic pathways within the myocardium [18].

Moreover, calcium homeostasis dysregulation has a chief role in DOX-induced cardiotoxicity. It was stated that the metabolism of DOX results in the formation of the toxic metabolite, doxorubicinol, which affects the sodium/potassium pump of the sarcolemma disrupting the sodium gradient needed for calcium to flow into the sarcolemma of the cardiomyocytes, resulting in a critical imbalance in the myocardial energetics and diminished systolic functions [17].

\subsection{Ischemic heart disease}

Ischemic heart disease, also called coronary arteries disease, is referred to the condition of the critical imbalance between myocardial blood supply and demand which is mainly resulted from the narrowing of the coronary arteries [19]. Although the narrowing can be caused by a blood clot or by constriction of the coronaries, most 
often it is caused by building up plaques, which is called atherosclerosis. Prolonged myocardial ischemia causes left ventricle enlargement, dilatation, and weakening resulting in the inhibition of the heart's ability for proper blood pumping. It also leads to a serious condition of cardiomyocytes death which is called myocardial infarction [4].

The dimeric protein complex, hypoxiainducible factor 1 (HIF-1), is the transcription factor of several target genes. It plays a major role in the body's compensation to hypoxic conditions and is considered as a main physiological regulator of vascularization, homeostasis, and anaerobic metabolism. It consists of an oxygen-regulated subunit, HIF-1 alpha, and a constitutively expressed subunit, HIF-1 beta [20].

Most importantly, hypoxia and inflammation have been sequentially bridged in ischemic heart disease. There is extensive crosstalk between the main hypoxic mediator, HIF-1, and the main inflammatory mediator, nuclear factor kappa B $(\mathrm{NF}-\mathrm{kB})$, including common activating stimuli, regulators, and targets. For instance, hypoxia has been identified as a common activator for both HIF-1 and NF- $\kappa \mathrm{B}$. It activates NF- $\kappa \mathrm{B}$ rapidly, within 5 to $30 \mathrm{~min}$, through the activation of the

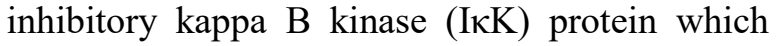
phosphorylates the inhibitory kappa $\mathrm{B}$ (IкB) protein resulting in the activation of NF- $\kappa \mathrm{B}$ [21]. Moreover, it was reported that some toll-like receptors (TLRs) are activated by the HIF-1 alpha subunit, resulting in the activation of NF$\kappa \mathrm{B}$ and increased pro-inflammatory cytokines production [22].

\subsection{Uncontrolled diabetes mellitus}

Uncontrolled diabetes mellitus leads to serious vascular disorders such as coronary arteries disease [23]. Several mechanisms influence the increased risk of cardiotoxicity development in diabetics, including changes in endothelial functions and the release of inflammatory mediators [24]. It was reported that type 2 diabetes mellitus, which is often associated with obesity, results in myocardial lipotoxicity that contributes to cardiomyocytes death and thus to cardiac dysfunction. Additionally, the heart biopsies of diabetic patients showed increased collagen depositions around intramural vessels and between myofibers, suggesting a mechanistic role for myocardial fibrosis in diabetic cardiotoxicity [25].

\subsection{Chronic alcohol consumption}

Long-term alcohol consumption is associated with the development of severe myocardial oxidative stress through the generation of huge amounts of free radicals resulting in severe cardiomyocytes damage [26]. Since mitochondria are major targets for free-radicals injury, chronic alcohol consumption leads to mitochondrial dysfunction either by disrupting the mitochondrial ultrastructure and/or depressing the indices of bioenergetics and oxidative phosphorylation. However, the damaged mitochondria are not only less bio energetically efficient, but they can also produce elevated amounts of ROS and initiate apoptotic degradation of the cardiomyocytes [27].

\subsection{Heavy metals intake}

Heavy metals such as arsenic, mercury, lead, cadmium, nickel, and aluminum are present at high concentrations in the earth's crust while they are present at very low concentrations in the human body [28]. Their major deleterious effects on humans are mainly through their accumulation in food, chiefly in vegetables that are grown on contaminated soil, resulting in chronic degenerative changes including cardiotoxicity, hepatotoxicity, and nervous system damage [29]. Heavy metals were reported to induce 
cardiotoxicity through the production of profuse amounts of free radicals within the myocardium, resulting in DNA damage, lipid peroxidation, and depletion of the cardiac antioxidant enzymes [30].

\subsection{Smoking}

Smoking is considered as a major risk factor for cardiovascular diseases [31]. It markedly upsurges the risk of acute cerebrovascular and coronary events, including stroke, myocardial infarction, and sudden cardiac death. It accelerates atherogenesis in the aorta, coronary arteries, carotid, and cerebral arteries, as well as peripheral circulation [32]. Furthermore, it was reported that smoking develops severe myocardial oxidative stress through the production of profuse amounts of ROS resulting in the damage of the cardiomyocytes and triggering the inflammatory and the apoptotic pathways [33].

\section{CARDIOTOXICITY COMPLICATIONS}

\subsection{Left ventricular failure}

A major predictor of mortality following cardiotoxicity is left ventricular dysfunction, as it results in pulmonary venous hypertension and pulmonary congestion [34]. The regulation of the ventricular shape, size, and function by neurohormonal, genetic, and mechanical factors is known as the ventricular remodeling process. It may be a physiological process that occurs during normal growth or pathological process due to cardiotoxicity [35].

Acute cardiac injury leads to a sudden elevation of the loading conditions that induce a unique remodeling pattern including the border zone of the damaged myocytes and then remotes to the non-damaged myocytes [36]. A cascade of biochemical intracellular signaling processes is triggered by the necrotic cardiomyocytes and the increased loading conditions resulting in the initiation and modulation of reparative changes such as cardiac hypertrophy and discrete collagen scars formation. Until the counterbalancing of distending forces by the tensile strength of the collagen scars occurs, ventricular remodeling may persist for weeks or months [34].

Furthermore, cardiotoxicity may result in major left ventricular abnormalities including left ventricular aneurysm, ventricular arrhythmia, ventricular septal defect, ventricular free wall rupture, and ventricular thrombus [37]. It is worth mentioning that as the extent of the left ventricle injury increases, the clinical manifestations of left ventricular failure becomes more common. Left ventricular failure commonly results in deleterious adverse outcomes including cardiac arrest and cardiac rupture [38].

\subsection{Cardiogenic shock}

Cardiogenic shock is considered as a low cardiac output state. It is the most severe clinical indication of left ventricular failure. The characteristic features of the low cardiac output state are the increased ventricular filling pressures, hypoperfusion of the vital organs, systemic hypotension, and cool extremities [39]. In this context, it was reported that more than $80 \%$ of myocardial ischemic patients are suffering from severe damage to the left ventricular myocardium in association with cardiogenic shock [40].

\subsection{Heart failure (HF)}

Heart failure (HF) is considered as a fatal outcome of cardiotoxicity. It is a state of compromised heart's ability to pump the blood throughout the body [41]. According to the affected part of the myocardium, there are different types of HF including left-sided, rightsided, and bilateral HF which may occur [42]. 


\section{CARDIOTOXICITY PATHOGENESIS AND MP CARDIOPROTECTIVE ROLES}

During the cardiac stressful conditions, sympathetic overactivation is considered as a crucial mechanism for providing short-term adaptation [43]. It results in catecholamine's excessive release, which in turn may affect the myocardial energy metabolism resulting in cardiotoxicity [44].

\subsection{Oxidative stress role}

Catecholamines produce excessive amounts of free radicals inducing myocardial damage, mainly due to their transformation into monochromes, which undergo redox cycling in mitochondria resulting in the production of profuse amounts of oxygen-derived free radicals [45]. Additionally, catecholamines-induced $\beta 1$ adrenoceptors activation has a predominant role in triggering oxidative stress through the production of free radicals [46]. The generated ROS in the myocardial tissue cause oxidative damage of membrane lipids, proteins, and deoxyribonucleic acid (DNA) leading to loss of myocardial membranes function and integrity. In this regard, the increased free radicals production in the myocardium results in increased levels of heart lipid peroxidation [4].

Lipid peroxidation is considered as a type of free radicals-mediated spreading of oxidative damage to the polyunsaturated fatty acid (PUFA) and this process comes to end through free radicals scavenging by antioxidants. It is considered as an important pathogenic event in cardiotoxicity. The accumulation of lipid hydroperoxides reflects the myocardial cellular damage and the lipid hydroperoxides can be used as oxidative stress biomarkers [47]. It was proven that the lipid peroxidation process results in the production of different stable compounds including $\alpha, \beta$-unsaturated aldehydes such as malondialdehyde (MDA) [48]. Moreover, the increased ROS production has been shown to initiate several processes that involved in atherogenesis, which is the main cause of myocardial tissue ischemic damage, including increased adhesion molecules expression, stimulated vascular smooth muscles proliferation, apoptosis within the endothelium, matrix metalloproteinases activation, and alteration of the vasomotor activity [49].

Endogenous antioxidants have a critical role in providing cytoprotective activities and preserving optimal cellular functions [50]. However, during severe cardiac oxidative stress conditions, endogenous antioxidants may be insufficient for providing appropriate cellular protection and maintenance of the appropriate functions of the cells [51]. The most effective antioxidant proteins include the antioxidant enzymes such as glutathione peroxidase (GPx), superoxide dismutase (SOD), and catalase (CAT) in addition to the non-enzymatic molecules such as reduced glutathione (GSH). The protection mechanisms of the antioxidant molecules include the prevention of oxidants generation, scavenging the free radicals, and inhibiting oxidant reactivity [50].

\subsection{Antioxidant activities of MP}

The antioxidant activity of MP has been investigated in several in-vivo models of chemical-induced toxicities. It has been verified against cyclophosphamide-induced cardiotoxicity in rats [14], lipopolysaccharide-induced acute lung injury in rats [52] and isoniazid and rifampicin-induced oxidative liver damage in mice [12] Collectively, MP antioxidant activity has been evidenced by the reduction of MDA and the raised enzymatic activities of GPx, SOD, and CAT, as summarized in Fig. 1. 


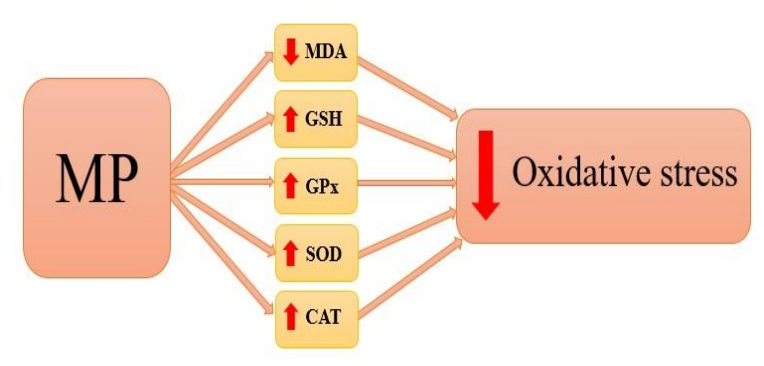

Fig. 1. Antioxidant activities of MP

MP, Methyl palmitate; MDA, Malondialdehyde; GSH, Reduced glutathione; GPx, Glutathione peroxidase; SOD, Superoxide dismutase; CAT, Catalase.

\subsection{Inflammatory cytokines role}

Inflammation is considered a key process in cardiotoxicity and various anti-inflammatory drugs proved a marked role in attenuating cardiotoxicity [53]. Inflammatory cytokines are considered fundamental mediators molecules for the inflammatory process. Generally, they are not expressed at a basal level within the myocardium, while their gene expression is elevated during myocardial injury [54].

In the case of cardiotoxicity, there are numerous harm signals which are known as damage-associated molecular patterns (DAMPs). They are considered as host-derived danger signals, that released in cases of tissue damage or metabolic disturbances [55]. As illustrated in Fig. 2, the release of DAMPs is known to activate inflammatory cascades initiated by toll-like receptors which in turn, activate intracellular adaptor proteins resulting in the activation of nuclear translocation of $\mathrm{NF}-\kappa \mathrm{B}$ leading to elevated transcription of pro-inflammatory genes and pro-inflammatory cytokines production [56]. Several studies proved the excessive release of pro-inflammatory cytokines such as interleukin 6 (IL-6), interleukin 1 (IL-1), tumor necrosis factor-alpha (TNF- $\alpha$ ) and cyclooxygenase-2 (COX-2) enzyme in case of cardiotoxicity, which is mainly depending on NF- $\mathrm{KB}$ activation [53, 57].

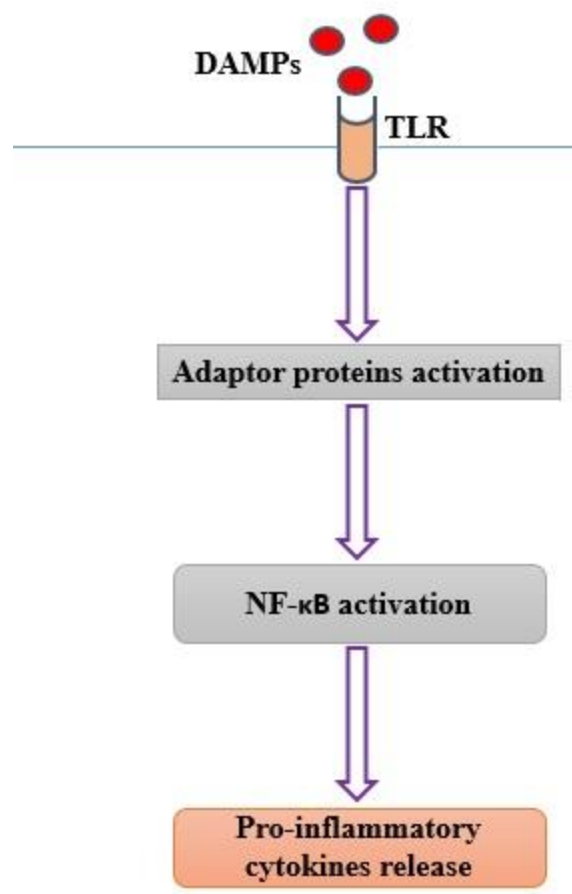

Fig. 2. Pro-inflammatory cytokines production in response to DAMPs

(DAMPs, Damage-associated molecular patterns; TLR, Toll like receptor; NF- $\kappa$ B, Nuclear factor kappa B).

The transcriptional factor NF- $\kappa \mathrm{B}$ consists of p65 and p50 subunits of the Rel protein family and is considered as a ubiquitous transcriptional factor that regulates the expression of numerous pro-inflammatory cytokines [58]. In quiescent cells, NF- $\kappa \mathrm{B}$ is prevented from activation by interacting with $\mathrm{I} \kappa \mathrm{B}$ in the cytoplasm. Stimulation of NF- $\kappa \mathrm{B}$ is considered as a response to various stimuli such as ROS, bacterial/viral antigens, and heavy metals [59]. In response to these stimuli, I $\mathrm{I} B$ is phosphorylated by $\mathrm{I} \kappa \mathrm{K}$ resulting in NF- $\kappa B$ activation and then its transportation from the cytoplasm into the nucleus, where it increases the expression of several pro-inflammatory cytokines such as IL-1, TNF- $\alpha$, and IL-6. Thus, NF- $\kappa$ B activation appears to play a critical role in the pathophysiology of endothelial dysfunction and cardiotoxicity [60]. 


\subsection{Anti-inflammatory activities of MP}

As illustrated in Fig. 3, the anti-inflammatory activities of MP were proven through the inhibition of the transcription factor NF- $\kappa \mathrm{B}$ and prevention of its translocation into the nucleus resulting in the reduced production of proinflammatory cytokines such as IL-1, TNF- $\alpha$, and IL-6 in addition to the elevated production of anti-inflammatory cytokines such as interleukin 10 (IL-10) [14].

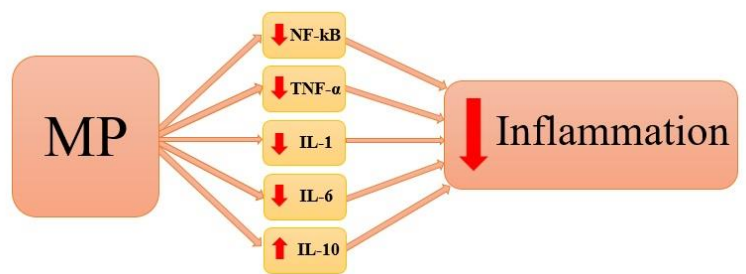

Fig. 3. Anti-inflammatory activities of MP

MP, Methyl palmitate; NF-кB, Nuclear factor kappa B; TNF- $\alpha$, Tumor necrosis factor alpha;

IL-1, Interleukin 1; IL-6, Interleukin 6; IL-10, Interleukin 10

Moreover, MP is also known to be one of the tolls like receptor 4 (TLR-4) antagonists, which were reported to be effective in the in-vivo murine model of transverse aortic constrictioninduced cardiac hypertrophy, as evidenced by the reduced production of pro-inflammatory cytokines and the augmented anti-inflammatory cytokines production [61]. It was stated that stimulation of TLR-4 leads to the recruitment of intracellular adaptor proteins to a signaling complex resulting in the activation of NF- $\kappa \mathrm{B}$ which elevates the of pro-inflammatory cytokines expression [56]. In this context, MP showed a cardioprotective effect against cyclophosphamide-induced cardiotoxicity in rats through the inhibition of the TLR-4/NF- $\kappa \mathrm{B}$ inflammatory pathway [14].

Additionally, MP also showed a major gastroprotective effect against ethanol-induced gastric mucosal injury due to its antiinflammatory activities evidenced by the inhibition of NF- $\kappa \mathrm{B}$ and mitogen-activated protein kinases (MAPKs) pathways [62]. Furthermore, MP exhibited anti-inflammatory effects against the lipopolysaccharide-stimulated phagocytic activity of rat peritoneal macrophages [63] and carrageenan-induced and croton oilinduced inflammation [13]. These antiinflammatory activities were mainly attributed to $\mathrm{NF}-\kappa \mathrm{B}$ inhibition as MP reduced the

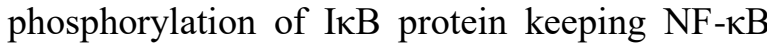
inhibited [64]. Also, MP showed robust neuroprotection in rat models of focal and global cerebral ischemia [65].

\subsection{Role of apoptosis}

Apoptosis is considered as a major determinant of the cardiomyocytes damage [66]. It is an energy-dependent, tightly regulated, and highly conserved form of cell death. Cell shrinkage, membrane blabbing, nuclear chromatin condensation, and chromosomal DNA fragmentation are the characteristic morphological changes of the apoptotic cells [67].

As shown in Fig. 4, initiation of apoptosis depends on two accepted and linked signaling pathways, the intrinsic and the extrinsic pathways, both converge on the same terminal or execution pathway [68].

As illustrated in Table 1, caspases are a family of protease enzymes that are considered as crucial apoptotic mediators. Caspase 3 is a frequently activated death protease that catalyzes the specific cleavage of many key cellular proteins [69]. The execution pathway starts with cleavage of caspase 3 resulting in degradation of cytoskeletal and nuclear proteins, DNA fragmentation, the formation of apoptotic bodies, expression of ligands for phagocytic cell receptors and finally uptake by phagocytic cells [67]. 
Table 1. Roles of different caspases

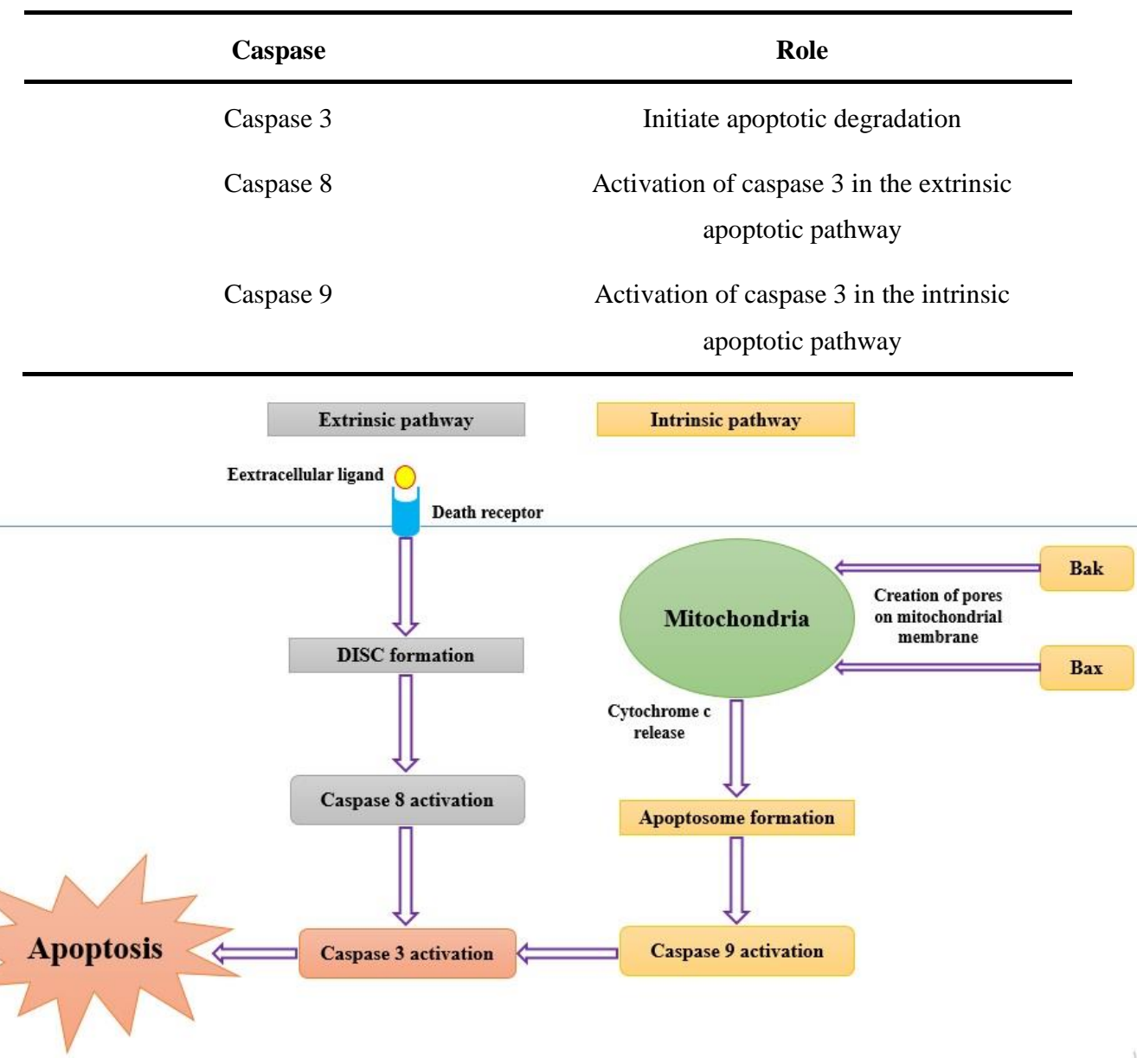

Fig. 4. Apoptotic pathways

Bak, Bcl-2 antagonist killer; Bax, Bcl-2-associated X; DISC, Death-inducing signaling complex

In the case of cardiotoxicity, as a result of the significantly elevated oxidative stress due to the massive ROS production, mitochondrial $\mathrm{Ca}^{2+}$ level upsurges beyond a threshold [70]. This mitochondrial $\mathrm{Ca}^{2+}$ overload triggers mitochondrial permeability transition, leading to mitochondrial swelling, loss of mitochondrial membrane potential and rupture of the outer membrane, and consequently, the release of cytochrome $\mathrm{c}$ which is normally located in the mitochondrion intermembrane space [71]. In the cytosol, cytochrome c binds further with the adaptor protein, apoptosis protease activation factor-1 (Apaf-1), forming apoptosome that recruits and activates the initiator caspase 9, which in turn activates caspase 3 that initiates the apoptotic degradation phase [72].

Most importantly, members of the B-cell lymphoma 2 (Bcl-2) family of proteins are the major regulators of the mitochondrial membrane potential including the pro-apoptotic proteins such as Bcl-2-associated $\mathrm{X}(\mathrm{Bax})$ and $\mathrm{Bcl}-2$ antagonist killer (Bak) and the anti-apoptotic proteins such as $\mathrm{Bcl}-2$ [73]. Cardiotoxicity is characterized by the elevated expression of the pro-apoptotic proteins and the reduced expression 
of anti-apoptotic proteins [74]. Translocation of the pro-apoptotic proteins to the outer mitochondrial membrane is believed to open the mitochondrial voltage-dependent anion channel leading to an increase of mitochondrial permeability. Besides, Bcl-2 has a major role in the maintenance of mitochondrial membrane integrity and stability [73].

On the other hand, it was reported that the binding of the extracellular ligands to cell-surface death receptors results in the formation of the death-inducing signaling complex (DISC) resulting in the activation of the extrinsic apoptotic pathway[75]. Members of tumor necrosis factor superfamily such as TNF- $\alpha$ have a predominant apoptotic role as their binding to TNF receptors initiates the extrinsic apoptotic pathway through the activation and cleavage of the initiator caspases such as caspase 8. Activated caspase 8 then directly cleaves and activates the effector caspases such as caspase 3. Effector caspases, in turn, cleave some different target proteins that play major roles in mediating apoptotic degradation [76].

\subsection{Anti-apoptotic activities of MP}

Indeed, MP exhibited anti-apoptotic activities against ethanol-induced gastric mucosal injury in rats [62] and against cyclophosphamide-induced cardiotoxicity in rats [14] as evidenced by the inhibited expression of the pro-apoptotic markers Bax and caspase 3 in addition to the increased expression of the anti-apoptotic protein $\mathrm{Bcl}-2$ in both experimental models.

\subsection{Role of fibrosis}

Cardiac fibrosis is a common finding in many cases of cardiotoxicity. It is an irreversible process that is recognized as a major cause of morbidity and mortality [77]. Characteristics of cardiac fibrosis include the net accumulation of extracellular matrix within the myocardium which is considered as an integral component of cardiotoxicity conditions [78]. Upon cardiac damage and stress conditions, various substances activate the fibroblasts and transdifferentiate them into myofibroblasts, which elevate the production of proteins that are deposited in the extracellular matrix making the myocardium stiffer [79]. These pathological changes result in cardiac electrical and structural abnormalities in addition to diminished cardiac functions that predispose patients to heart failure [80].

\subsection{Anti-fibrotic activities of MP}

As well, MP was further reported to have antifibrotic activities as evidenced by its ability of protection against carbon tetrachloride-induced liver fibrosis. It inhibited the expression of the fibrotic markers hydroxyproline and alphasmooth muscle actin ( $\alpha$-SMA) in carbon tetrachloride-induced liver fibrosis in rats [81]. Besides, the epidural fibrosis formation was decreased by MP through diminishing the functions of inflammatory cells such as fibroblasts, macrophages, and neutrophils [15]. Furthermore, MP showed anti-fibrotic activity in a rat model of silica-induced lung fibrosis [82]. The anti-fibrotic activities of MP in these experimental models potentiate that it can possess a cardioprotective activity through the prevention of cardiac fibrosis.

\subsection{Vasodilatation activity of MP}

Previous studies stated that there are identical chemical and pharmacological properties between MP and the endogenous perivascular adipose tissue-releasing factor (PVATRF) which regulates the vascular tone, suggesting that the PVATRF is MP, which plays a crucial role in regulating the vascular tone through opening the potassium channels $(\mathrm{Kv})$ on the vascular smooth muscle to induce vasodilatation. [83]. Additionally, it was reported that MP induces endothelium-independent aortic relaxation through its action on $\mathrm{Kv}$ of the smooth muscle 
cells inducing vasodilatation. This was proven by using a superfusion bioassay cascade technique with rat isolated retina as a donor tissue and rat aortic ring as a detector tissue [8]. In this regard, it was stated that vasodilating compounds have a beneficial role in various cardiovascular diseases by decreasing cardiac load preventing further cardiotoxicity [84].

\subsection{Miscellaneous cytoprotective activities of MP}

Furthermore, MP also proved a reduction/inhibition against chemically-induced hepatotoxicity in rats, which can be induced by 1,2 dichlorobenzene, monoethyl hexyl phthalate, and 4-chloro-6-(2,3-xylidino) pyrimidinylthioacetic acid [85-87]. Additionally, pretreatment with MP prevented graft failure and elevated the survival of orthotopic liver transplantation in rats [88]. These therapeutic effects are based on the MP-inhibitory effect on kupffer cell functions [89]. As well, the protective effect of MP had been also proven against non-alcoholic steatohepatitis through the induction of peroxisome proliferator-activated receptor alpha (PPAR- $\alpha$ ) pathway in a mice model of methionine choline-deficient diet-induced steatohepatitis [5]. In addition, MP showed major protection against sepsis in mice [90]. Finally, these different cytoprotective activities of MP potentiate that it can possess a cardioprotective role.

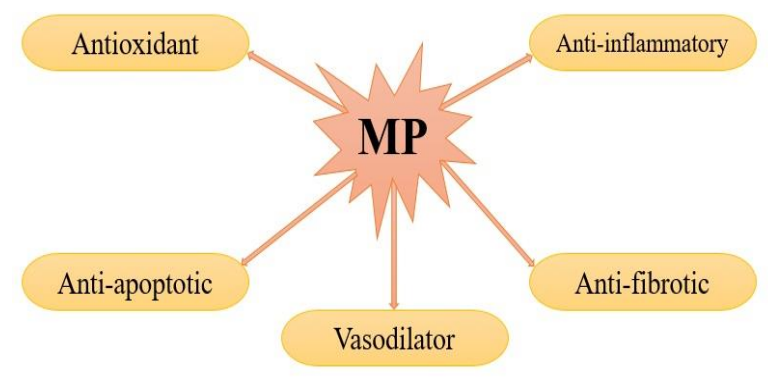

Fig. 5. Cardioprotective activities of MP MP, Methyl palmitate.

\section{CONCLUSION}

This review article highlights the cardioprotective activities of MP. The main mechanisms underlying these activities mainly attributed to the antioxidant, anti-inflammatory, anti-apoptotic, anti-fibrotic, and vasodilatation properties of MP, as depicted in Fig. 5.

\section{DECLARATIONS}

\section{Ethics approval and consent to participate}

Not applicable.

\section{Consent to publish}

Not applicable.

\section{Availability of data and materials}

All data generated or analyzed during this study are included in this published article in the main manuscript.

\section{Competing interests}

The authors declare that no competing interests exist.

\section{Funding statement}

No funding source was received.

\section{Authors' contributions}

The manuscript was drafted and written by A.B.H. The other authors: E.M.M., W.M.E, Y.A., and S.S.A. have provided comments and contributed to revising the manuscript. All authors have read and approved the final manuscript.

\section{Acknowledgment}

The authors would like to acknowledge all colleagues in the Pharmacology and Toxicology Department, Faculty of Pharmacy, Ain Shams University, and Pharmacology, Toxicology and Biochemistry Department, Faculty of Pharmaceutical Sciences, and Pharmaceutical Industries, Future University in Egypt. 


\section{List of abbreviations}

Apaf-1, Apoptosis protease activation factor1; Bak, Bcl-2 antagonist killer; Bax, Bcl-2associated X; Bcl-2, B-cell lymphoma 2; CAT, Catalase; COX 2, cyclooxygenase-2; DAMPs, Damage-associated molecular patterns; DISC, Death-inducing signaling complex; DNA, Deoxyribonucleic acid; Doxorubicin, DOX; GPx, Glutathione peroxidase; GSH, Reduced glutathione; HIF-1, Hypoxia-inducible factor 1;

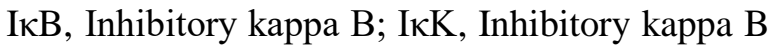
kinase; IL-1, Interleukin 1; IL-6, Interleukin 6; IL-10, Interleukin 10; Kv, Potassium channels; MAPKs, Mitogen-activated protein kinases; MDA, Malondialdehyde; MP, Methyl palmitate; NF-кB, Nuclear factor-kappa B; PPAR- $\alpha$, Peroxisome proliferator-activated receptor alpha; PUFA, Polyunsaturated fatty acid; PVATRF, Perivascular adipose tissue-releasing factor; ROS, Reactive oxygen species; $\alpha$-SMA, Alphasmooth muscle actin; SOD, Superoxide dismutase; TLR-4, Toll-like receptor 4; TNF- $\alpha$, Tumor necrosis factor-alpha.

\section{REFERENCES}

1. Dhyani N, Parveen A, Siddiqi A, Hussain ME, Fahim M. Cardioprotective Efficacy of Coriandrum sativum (L.) seed extract in heart failure rats through modulation of endothelin receptors and antioxidant potential. J Diet Suppl 2020; 17(1): 13-26. DOI: 10.1080/19390211.2018.1481483.

2. Acharya S, Siddiqui A, Anwar S, Habib S, Maroun R. Lithium-induced cardiotoxicity: a rare clinical entity. Cureus 2020; 12(3): 72-86. DOI: $10.7759 /$ cureus. 7286 .

3. Gupta A, Shukla P, Ashwalayan V. Clinical assessment of cardiovascular disorders by cardiac biomarkers. Int J Pharm Sci Rev Res 2014; 29(1): 87-94.

4. Yu Y, Jin L, Zhuang Y, Hu Y, Cang J, Guo K. Cardioprotective effect of rosuvastatin against isoproterenol-induced myocardial infarction injury in rats. Int J Mol Med 2018; 41(6): 35093516. DOI: 10.3892/ijmm.2018.3572.

5. Zhang L, Li HX, Pan WS, Khan FU, Qian C, Qi-Li FR, et al. Administration of methyl palmitate prevent non-alcoholic steatohepatitis (NASH) by induction of PPAR- $\alpha$. Biomed Pharmacother 2019; 111: 99-108. DOI: 10.1016/j.biopha.2018.12.059.

6. Ndiaye EHI, Habrioux M, Coutinho JA, Paredes Mr, Daridon JL. Speed of sound, density, and derivative properties of ethyl myristate, methyl myristate, and methyl palmitate under high pressure. J Chem Eng Data 2013; 58(5): 13711377. DOI: $10.1021 /$ je400122k.

7. Lin HW, Liu CZ, Cao D, Chen PY, Chen MF, Lin SZ, et al. Endogenous methyl palmitate modulates nicotinic receptor-mediated transmission in the superior cervical ganglion. Proc Natl Acad Sci 2008; 105(49): 1952619531. DOI: $10.1073 /$ pnas.0810262105.

8. Lee YC, Chang HH, Liu CH, Chen MF, Chen PY, Kuo JS, et al. Methyl palmitate: a potent vasodilator released in the retina. Invest Ophthalmol Vis Sci 2010; 51(9): 4746-4753. DOI: 10.1167/iovs.09-5132.

9. Lin Y, Xu F, Li Z, Yang L, Li B, Deng G. Analysis of volatile composition in the perfume of ficus carica lam. produced by fermentation. Biotechnology 2005; 15: 54-56.

10. Wang Y, Wang H, Shen Z, Zhao L, Clarke S, Sun J, et al. Methyl palmitate, an acaricidal compound occurring in green walnut husks. J Econ Entomol 2009; 102(1): 196-202. DOI: 10.1603/029.102.0128.

11. Saravanan K, Tyagi B, Shukla RS, Bajaj HC. Solvent-free synthesis of methyl palmitate over sulfated zirconia solid acid catalyst. Fuel 2016; 165: 298-305. DOI: 10.1016/j.fuel.2015.10.043.

12. Kalemci S, Sarihan A, Dere Y. Methyl 
palmitate protection against isoniazid/rifampisicin-induced oxidative liver damage. Iran Red Crescent Med J 2018; 20(5): e60819. DOI: 10.5812/ircmj.60819.

13. Saeed NM, El-Demerdash E, Abdel-Rahman HM, Algandaby MM, Al-Abbasi FA, AbdelNaim AB. Anti-inflammatory activity of methyl palmitate and ethyl palmitate in different experimental rat models. Toxicol Appl Pharmacol 2012; 264(1): 84-93. DOI: 10.1016/j.taap.2012.07.020.

14.El-Agamy DS, Elkablawy MA, Abo-Haded HM. Modulation of cyclophosphamide-induced cardiotoxicity by methyl palmitate. Cancer Chemother Pharmacol 2017; 79(2): 399-409. DOI: $10.1007 / \mathrm{s} 00280-016-3233-1$.

15. Kizilay Z, Kahraman Cetin N. Effect of methyl palmitate on the formation of epidural fibrosis in an experimental epidural fibrosis model. J Invest Surg 2018; 31(6): 469-474. DOI: 10.1080/08941939.2017.1356403.

16. Ewer MS, Ewer SM. Cardiotoxicity of anticancer treatments. Nat Rev Cardiol 2015; 12(9): 547-558. DOI: 10.1038/nrcardio.2015.65.

17.dos Santos DS, dos Santos Goldenberg RC. Doxorubicin-induced cardiotoxicity: from mechanisms to development of efficient therapy. In: Tan W, editor. Cardiotoxicity. London: IntechOpen; 2018, p. 3-19.

18. Abushouk AI, Ismail A, Salem AMA, Afifi AM, Abdel-Daim MM. Cardioprotective mechanisms of phytochemicals against doxorubicin-induced cardiotoxicity. Biomed Pharmacother 2017; 90: 935-946. DOI: 10.1016/j.biopha.2017.04.033.

19. Yang L, Xie P, Wu J, Yu J, Yu T, Wang H, et al. Sevoflurane postconditioning improves myocardial mitochondrial respiratory function and reduces myocardial ischemia-reperfusion injury by up-regulating HIF-1. Am J Transl Res 2016; 8(10): 4415-4424.

20. Ziello JE, Jovin IS, Huang Y. Hypoxiainducible factor (HIF)-1 regulatory pathway and it's potential for therapeutic intervention in malignancy and ischemia. Yale J Biol Med 2007; 80(2): 51-60.

21. D'Ignazio L, Rocha S. Hypoxia-induced NF-кB. Cells 2016; 5(1): 10-17. DOI: 10.3390/cells5010010.

22.Tafani M, Pucci B, Russo A, Schito L, Pellegrini L, Perrone GA, et al. Modulators of HIF $1 \alpha$ and NFkB in cancer treatment: is it a rational approach for controlling malignant progression?. Front Pharmacol 2013; 4: 13-24. DOI: 10.3389/fphar.2013.00013.

23. Domingueti CP, Dusse LMSA, das Graças Carvalho M, de Sousa LP, Gomes KB, Fernandes AP. Diabetes mellitus: the linkage between oxidative stress, inflammation, hypercoagulability, and vascular complications. J Diabetes Complications 2016; 30(4): 738745 .

24.Larsson SC, Wallin A, Håkansson N, Stackelberg O, Bäck M, Wolk A. Type 1 and type 2 diabetes mellitus and incidence of seven cardiovascular diseases. Int J Cardiol 2018; 262: 66-70.

25. Boudina S, Abel ED. Diabetic cardiomyopathy, causes, and effects. Rev Endocr Metab Dis 2010; 11(1): 31-39. DOI: 10.1007/s11154-0109131-7.

26. Fernández SJ. The Effects of ethanol on the heart: alcoholic cardiomyopathy. Nutrients 2020; 12(2): 572-589. DOI: 10.3390/nu12020572.

27.Piano MR, Phillips SA. Alcoholic cardiomyopathy: pathophysiologic insights. Cardiovasc Toxicol 2014; 14(4): 291-308. DOI: 10.1007/s12012-014-9252-4.

28. Islam SJ, Manna P, Unni B, Kailta J. Higher concentrations of heavy metals impair antioxidant defense mechanism and growth response of muga silkworm, Antheraea assamensis (lepidoptera: saturniidae). J Entomol Zool Stud 2019; 7(2): 715-724. 
29. Alissa EM, Ferns GA. Heavy metal poisoning and cardiovascular disease. J Toxicol 2011; 2011: 1-21. DOI: $10.1155 / 2011 / 870125$.

30. Al-Taher AY, Morsy MA, Rifaai RA, Zenhom NM, Abdel-Gaber SA. Paeonol attenuates methotrexate-induced cardiac toxicity in rats by inhibiting oxidative stress and suppressing TLR4-induced NF- $\kappa \mathrm{B}$ inflammatory pathway. Mediators Inflamm 2020; 2020: 1-10. DOI: $10.1155 / 2020 / 8641026$.

31.Ek A, Ekblom Ö, Hambraeus K, Cider $\AA$, Kallings LV, Börjesson M. Physical inactivity and smoking after myocardial infarction as predictors for readmission and survival: results from the swedeheart-registry. Clin Res Cardiol 2019; 108(3): 324-332. DOI: 10.1007/s00392018-1360-х.

32. Benowitz NL, Burbank AD. Cardiovascular toxicity of nicotine: implications for electronic cigarette use. Trends Cardiovasc Med 2016; 26(6): 515-523. DOI: 10.1016/j.tcm.2016.03.001.

33. Kamceva G, Arsova-Sarafinovska Z, Ruskovska T, Zdravkovska M, Kamceva-Panova L, Stikova E. Cigarette smoking and oxidative stress in patients with coronary artery disease. Maced J Med Sci 2016; 4(4): 636-640. DOI: 10.3889/oamjms.2016.117.

34. Cha MJ, Lee JH, Jung HN, Kim Y, Choe YH, Kim SM. Cardiac magnetic resonance-tissue tracking for the early prediction of adverse left ventricular remodeling after ST-segment elevation myocardial infarction. Int J Cardiovas Imag 2019; 35(11): 2095-2102. DOI: 10.1007/s10554-019-01659-w.

35. Roever L, Palandri Chagas AC. Cardiac remodeling: new insights in physiological and pathological adaptations. Front Physiol 2017; 8: 751-753. DOI: 10.3389/fphys.2017.00751.

36. Azevedo PS, Polegato BF, Minicucci MF, Paiva SA, Zornoff LA. Cardiac remodeling: concepts, clinical impact, pathophysiological mechanisms, and pharmacologic treatment. Arq Bras Cardiol 2016; 106(1): 62-69. DOI: 10.5935/abc.20160005

37. Bahit MC, Kochar A, Granger CB. Postmyocardial infarction heart failure. JACC Heart Fail 2018; 6(3): 179-186. DOI: 10.1016/j.jchf.2017.09.015.

38. Choi JY, Cha J, Jung JM, Seo WK, Oh K, Cho $\mathrm{KH}$, et al. Left ventricular wall motion abnormality is associated with cryptogenic stroke. Int J Stroke 2020; 15(2): 188-196. DOI: $10.1177 / 1747493019834181$.

39. Rodriguez MML, Guerrero CJV, Garzon JCA, Ortiz DFHPA, Avila AFS, Padilla JDV. Cardiogenic shock. Interv Cardiol 2019; 11(6): 123-126.

40. Reynolds HR, Hochman JS. Cardiogenic shock: Current concepts and improving outcomes. Circulation 2008; 117(5): 686-697. DOI: 10.1161/circulationaha.106.613596.

41. Giustino G, Redfors B, Brener SJ, Kirtane AJ, Généreux P, Maehara A, et al. Correlates and prognostic impact of new-onset heart failure after ST-segment elevation myocardial infarction treated with primary percutaneous coronary intervention: insights from the infuseAMI trial. Eur Heart J Acute Cardiovasc Care 2018; 7(4): 339-347. DOI: $10.1177 / 2048872617719649$.

42.Bauer A, Esmaeili A, deRosa R, Voelkel NF, Schranz D. Restrictive atrial communication in right and left heart failure. Transl Pediatr 2019; 8(2): 133-139. DOI: 10.21037/tp.2019.04.03.

43. Olmati F, Petramala L, Bisogni V, Concistré A, Saracino V, Oliviero G, et al. A rare case report of hypertrophic cardiomyopathy induced by the catecholamine-producing tumor. Medicine 2018; 97(50): e13369. DOI: 10.1097/MD.0000000000013369.

44. Todd GL. Cardiotoxicity of catecholamines. In: Acosta D, editor. Cellular and molecular 
toxicology and in vitro toxicology. Florida: CRC Press; 2017, p. 91-128.

45.Liaudet L, Calderari B, Pacher P. Pathophysiological mechanisms of catecholamine and cocaine-mediated cardiotoxicity. Heart Fail Rev 2014; 19(6): 815824. DOI: 10.1007/s10741-014-9418-y.

46. Jagadeesh GS, Meeran MFN, Selvaraj P. Activation of $\beta 1$-adrenoceptor triggers oxidative stress-mediated myocardial membrane destabilization in isoproterenol-induced myocardial infarcted rats: 7-hydroxycoumarin and its counteraction. Eur J Pharmacol 2016; 777: 70-77. DOI: 10.1002/jbt.21770.

47. Kagan VE. Lipid Peroxidation in Biomembranes. Florida: CRC Press; 2018.

48. Morales M, Munné BS. Malondialdehyde: facts and artifacts. Plant Physiol 2019; 180(3): 12461250. DOI: $10.1104 /$ pp.19.00405.

49. Kattoor AJ, Pothineni NVK, Palagiri D, Mehta JL. Oxidative stress in atherosclerosis. Curr Atheroscler Rep 2017; 19: 42-52. DOI: 10.1007/s11883-017-0678-6.

50. Aguilar TAF, Navarro BCH, Perez JAM. Endogenous antioxidants: a review of their role in oxidative stress. In: Gonzalez JA, González Á, Santillan EO, editors. A master regulator of oxidative stress-the the transcription factor nrf2. London: IntechOpen; 2016, p. 3-20.

51.Kurutas EB. The importance of antioxidants which play the role in cellular response against oxidative/nitrosative stress: current state. Nutr J 2015; 15: 71-92. DOI: 10.1186/s12937-0160186-5.

52. Ammar EM, Sharawy MH, Shalaby AA, ElAgamy DS. Effects of methyl palmitate and lutein on LPS-induced acute lung injury in rats. World J Respirol 2013; 3(2): 20-28. DOI: 10.5320/wjr.v3.i2.20.

53. Quagliariello V, Vecchione R, Coppola C, Di Cicco C, De Capua A, Piscopo G, et al.
Cardioprotective effects of nanoemulsions loaded with anti-inflammatory nutraceuticals against doxorubicin-induced cardiotoxicity. Nutrients 2018; 10(9): 1304-1326. DOI: 10.3390/nu10091304.

54.Daskalopoulos EP, Hermans KC, van Delft L, Altara R, Blankesteijn WM. The role of inflammation in myocardial infarction. In: Blankesteijn W, Altara R, editors. Inflammation in heart failure. Amsterdam: Elsevier; 2015, p. 39-65.

55. Gregersen I, Halvorsen B. Inflammatory mechanisms in atherosclerosis. In: Gonzalez JA, Santillan EO, editors. Cardiology and cardiovascular medicine. London: IntechOpen; 2017, p. 3-18.

56. Yang Y, Lv J, Jiang S, Ma Z, Wang D, Hu W, et $a l$. The emerging role of toll-like receptor 4 in myocardial inflammation. Cell Death Dis 2017; 7(5): e2234. DOI: 10.1038/cddis.2016.140.

57.Janahmadi Z, Nekooeian AA, Moaref AR, Emamghoreishi M. Oleuropein offers cardioprotection in rats with acute myocardial infarction. Cardiovasc Toxicol 2015; 15(1): 6168. DOI: 10.1007/s12012-014-9271-1.

58. Panday A, Inda ME, Bagam P, Sahoo MK, Osorio D, Batra S. Transcription factor NF- $\kappa B$ : an update on intervention strategies. Arch Immunol Ther Exp 2016; 64(6): 463-483. DOI: 10.1007/s00005-016-0405-y.

59. Brasier AR. The NF- $\kappa \mathrm{B}$ regulatory network. Cardiovasc Toxicol 2006; 6(2): 111-130. DOI: 10.1385/CT:6:2:111.

60. Hoffmann A, Natoli G, Ghosh G. Transcriptional regulation via the NF- $\mathrm{KB}$ signaling module. Oncogene 2006; 25(51): 6706-6716. DOI: 10.1038/sj.onc.1209933.

61. Ehrentraut H, Weber C, Ehrentraut S, Schwederski M, Boehm O, Knuefermann P, et al. The toll-like receptor 4-antagonist eritoran reduces murine cardiac hypertrophy. Eur J Heart Fail 2011; 13(6): 602-610. DOI: 


\subsection{3/eurjhf/hfr035.}

62. Arab HH, Salama SA, Eid AH, Kabel AM, Shahin NN. Targeting MAPKs, NF- $\kappa$ B, and PI3K/AKT pathways by methyl palmitate ameliorates ethanol-induced gastric mucosal injury in rats. J Cell Physiol 2019; 234(12): 22424-22438. DOI: 10.1002/jcp.28807.

63. Sarkar S, Khan MF, Kaphalia BS, Ansari G. Methyl palmitate inhibits the lipopolysaccharide-stimulated phagocytic activity of rat peritoneal macrophages. J Biochem Mol Toxicol 2006; 20(6): 302-308. DOI: $10.1002 /$ jbt.20150.

64.El-Demerdash E. Anti-inflammatory and antifibrotic effects of methyl palmitate. Toxicol Appl Pharmacol 2011; 254(3): 238-244. DOI: 10.1016/j.taap.2011.04.016.

65.Lin HW, Saul I, Gresia VL, Neumann JT, Dave KR, Perez-Pinzon MA. Fatty acid methyl esters and solutol HS 15 confer neuroprotection after focal and global cerebral ischemia. Transl Stroke Res 2014; 5(1): 109-117. DOI: 10.1007/s12975-013-0276-z.

66. Ma Y, Yang L, Ma J, Lu L, Wang X, Ren J, et al. Rutin attenuates doxorubicin-induced cardiotoxicity via regulating autophagy and apoptosis. Biochim Biophys Acta 2017; 1863(8): 1904-1911. DOI: 10.1016/j.bbadis.2016.12.021.

67. Nagata S. Apoptosis and clearance of apoptotic cells. Annu Rev Immunol 2018; 36: 489-517. DOI: $\quad$ 10.1146/annurev-Immunol-042617053010 .

68. Derakhshan A, Chen Z, Van WC. Therapeutic small molecules target inhibitor of apoptosis proteins in cancers with the deregulation of extrinsic and intrinsic cell death pathways. Clin Cancer Res 2017; 23(6): 1379-1387. DOI: 10.1158/1078-0432.CCR-16-2172.

69.Julien O, Wells JA. Caspases and their substrates. Cell Death Differ 2017; 24(8): 1380-
1389. DOI: $10.1038 / \mathrm{cdd} .2017 .44$.

70.Liang J, Cao R, Wang X, Zhang Y, Wang P, $\mathrm{Gao} \mathrm{H}$, et al. Mitochondrial PKM2 regulates oxidative stress-induced apoptosis by stabilizing Bcl2. Cell Res 2017; 27(3): 329-351. DOI: 10.1038/cr.2016.159.

71.Lee JY, Lim W, Ham J, Kim J, You S, Song G. Ivermectin induces apoptosis of porcine trophectoderm and uterine luminal epithelial cells through loss of mitochondrial membrane potential, mitochondrial calcium ion overload, and reactive oxygen species generation. Pestic Biochem Physiol 2019; 159: 144-153. DOI: 10.1016/j.pestbp.2019.06.009.

72. Nicholson DW, Thornberry NA. Life and death decisions. Science 2003; 299(5604): 214-215. DOI: $10.1126 /$ science. 1081274.

73.Edlich F. BCL-2 proteins and apoptosis: recent insights and unknowns. Biochem Biophys Res Commun 2018; 500(1): 26-34. DOI: 10.1016/j.bbrc.2017.06.190.

74. Subbarao RB, Ok SH, Lee SH, Kang D, Kim EJ, Kim JY, et al. Lipid emulsion inhibits the late apoptosis/cardiotoxicity induced by doxorubicin in rat cardiomyoblasts. Cells 2018; 7(10): 144159. DOI: $10.3390 /$ cells7100144.

75.Hongmei Z. Extrinsic and intrinsic apoptosis signal pathway review. In: Ntuli $\mathrm{T}$, editor. Apoptosis and medicine. London: InTechOpen; 2012, p. 2-21.

76. Kuo WT, Shen L, Zuo L, Shashikanth N, Ong MLDM, Wu L, et al. Inflammation-induced occludin downregulation limits epithelial apoptosis by suppressing caspase-3 expression. Gastroenterology 2019; 157(5): 1323-1337. DOI: $10.1053 /$ j.gastro.2019.07.058.

77. Murtha LA, Schuliga MJ, Mabotuwana NS, Hardy SA, Waters DW, Burgess JK, et al. The processes and mechanisms of cardiac and pulmonary fibrosis. Front Physiol 2017; 8: 777791. DOI: 10.3389/fphys.2017.00777. 
78. Wang X, Jin H, Jiang S, Xu Y. MicroRNA-495 inhibits the high glucose-induced inflammation, differentiation, and extracellular matrix accumulation of cardiac fibroblasts through the downregulation of NOD1. Cell Mol Biol Lett 2018; 23(1): 23-35. DOI: 10.1186/s11658-0180089-x.

79.Levick SP, Soto-Pantoja DR, Bi J, Hundley WG, Widiapradja A, Manteufel EJ, et al. Doxorubicin-induced myocardial fibrosis involves the neurokinin-1 receptor and direct effects on cardiac fibroblasts. Heart Lung Circ 2019; 28(10): 1598-1605. DOI: 10.1016/j.hlc.2018.08.003.

80.Heymans S, González A, Pizard A, Papageorgiou AP, López-Andrés N, Jaisser F, et al. Searching for new mechanisms of myocardial fibrosis with diagnostic and/or therapeutic potential. Eur J Heart Fail 2015; 17(8): 764-771. DOI: 10.1002/ejhf.312.

81. Mantawy EM, Tadros MG, Awad AS, Hassan DA, El-Demerdash E. Insights antifibrotic mechanism of methyl palmitate: impact on nuclear factor kappa B and proinflammatory cytokines. Toxicol Appl Pharmacol 2012; 258(1): 134-144. DOI: 10.1016/j.taap.2011.10.016.

82. Sharawy MH, El-Agamy DS, Shalaby AA, Ammar E-SM. Protective effects of methyl palmitate against silica-induced pulmonary fibrosis in rats. Int Immunopharmacol 2013; 16(2): $\quad 191-198 . \quad$ DOI: 10.1016/j.intimp.2013.04.007.

83. Lee YC, Chang HH, Chiang CL, Liu CH, Yeh JI, Chen MF, et al. Role of perivascular adipose tissue-derived methyl palmitate in vascular tone regulation and pathogenesis of hypertension. Circulation 2011; 124(10): 1160-1171. DOI: 10.1161/circulationaha.111.027375.

84.Tarkin JM, Kaski JC. Vasodilator therapy: nitrates and nicorandil. Cardiovasc Drugs Ther 2016; 30(4): 367-378. DOI: 10.1007/s10557- 016-6668-z.

85. Gunawardhana L, Mobley S, Sipes I. Modulation of 1, 2-dichlorobenzene hepatotoxicity in the Fischer-344 rat by a scavenger of superoxide anions and an inhibitor of kupffer cells. Toxicol Appl Pharmacol 1993; 119(2): 205-213. DOI: 10.1006/taap.1993.1061.

86. Rose ML, Rusyn I, Bojes HK, Belyea J, Cattley RC, Thurman RG. Role of Kupffer cells and oxidants in signaling peroxisome proliferatorinduced hepatocyte proliferation. Mutat ResFund Mol Mech 2000; 448(2): 179-192. DOI: 10.1.1.714.5947.

87. Sipes I, El Sisi A, Sim W, Mobley S, Earnest D. Reactive oxygen species in the progression of $\mathrm{CCl}$ 4-induced liver injury. In: Witmer CM, Snyder RR, Jollow DJ, Kalf GF, Kocsis JJ, Sipes IG, editors. Biological reactive intermediates IV. Berlin: Springer; 1991, p. 489497.

88. Marzi I, Cowper K, Takei Y, Lindert K, Lemasters JJ, Thurman RG. Methyl palmitate prevents kupffer cell activation and improves survival after orthotopic liver transplantation in the rat. Transpl Int 1991; 4(1): 215-220. DOI: 10.1111/j.1432-2277.1991.tb01983.x.

89. Cai P, Kaphalia B, Ansari G. Methyl palmitate: inhibitor of phagocytosis in primary rat Kupffer cells. Toxicology 2005; 210(2): 197-204. DOI: 10.1016/j.tox.2005.02.001.

90. Villa P, Demitri M, Meazza C, Sironi M, Gnocchi P, Ghezzi P. Effects of methyl palmitate on cytokine release, liver injury, and survival in mice with sepsis. Eur Cytokine Netw 1996; 7(4): 765-769. 